\title{
0 Efeito de EPDM Modificado com Grupos Mercapto ou Tioacetato na Cinética de Vulcanização de Misturas NR/EPDM
}

\author{
Alex S. Sirqueira, Bluma G. Soares \\ Instituto de Macromoléculas, UFRJ
}

Resumo: A cinética de vulcanização de misturas envolvendo borracha natural (NR) e copolímero de etileno-propileno-dieno (EPDM) na proporção 70:30 \% em peso foi estudada em função da compatibilização. Para esse estudo foram utilizados 2,5 phr de EPDM funcionalizado com grupos tioacetato (EPDMTA) ou mercaptana (EPDMSH). O estudo foi realizado em reômetro de disco oscilatório (curômetro). Ambos os copolímeros funcionalizados atuaram como aceleradores do processo de vulcanização das misturas NR/EPDM (70:30) na presença de enxofre e CBS, sendo EPDMSH mais efetivo do que EPDMTA pelo modelo de Chough e Chang. Entretanto, ao utilizar o modelo de Coran, observou-se que EPDMTA e EPDMSH atuam em distintas etapas da vulcanização: EPDMSH atua preferencialmente no início do processo de vulcanização enquanto que EPDMTA aumenta a velocidade da formação das reticulações propriamente ditas. Além disso, EPDMTA diminuiu consideravelmente a tendência à degradação de reticulações, indicada pela menor taxa de reversão.

Palavras-chave: Misturas elastoméricas, borracha natural, cinética de vulcanização, compatibilização.

\section{The Effect of Mercapto- and Thioacetate-Modified EPDM on the Vulcanization Kinetics of NR/EPDM Blends}

Abstract: Vulcanization kinetics for natural rubber (NR)/ ethylene-propylene-diene rubber (EPDM) blends was studied as a function of the compatibilization, by using an oscillatory disk rheometer. For this study, $2.5 \mathrm{phr}$ of thioacetate- (EPDMTA) or mercapto-modified EPDM (EPDMSH) were employed in blends vulcanized with sulfur/CBS curing system. Both functionalized copolymers increased the curing velocity of the blends, but the higher overall rate was achieved with EPDMSHmodified systems, using the Chough and Chang model. However, when using the Coran model, we observed that they act in distinct vulcanization steps: EPDMSH acts preferentially at the beginning of the curing process whereas EPDMTA increases the rate of the crosslink formation. In addition, EPDMTA decreased the tendency for crosslink decomposition, as indicated by the lower value of reversion degree.

Keywords: Elastomer blends, natural rubber, vulcanization kinetics, compatibilization.

\section{Introdução}

Misturas de borracha natural (NR) com copolímeros de etileno-propileno-dieno (EPDM) têm sido largamente estudadas, visando o desenvolvimento de materiais elastoméricos com maior resistência ao envelhecimento. Entretanto, as propriedades mecânicas dessas misturas podem ser prejudicadas devido à incompatibilidade entre os componentes ${ }^{[1,2]}$. Várias estratégias têm sido relatadas na literatura para contornar esses problemas e incluem a adição de um terceiro componente de baixa massa molar ${ }^{[3]}$, a incorporação de moléculas de acelerador de vulcanização na cadeia de $E P D M^{[4]}$, e a utilização de $E P D M$ funcionalizado com grupos derivados do anidrido maleico ${ }^{[5-7]}$.

Trabalhos recentes desenvolvidos pelo nosso grupo de pesquisa também relatam a eficiência do EPDM modificado com grupos mercaptana (EPDMSH), em melhorar as propriedades mecânicas de misturas de $N R / E P D M^{[8-11]}$. O efeito compatibilizante desse copolímero funcionalizado baseia-se na capacidade dos grupos mercaptana em reagir com as ligações duplas do elastômero mais insaturado, a borracha natural, resultando em forte interação interfacial. Grupos mercaptana incorporados no copolímero de etileno- acetato de vinila (EVA) também atuaram como agentes interfaciais em misturas de $N R / \mathrm{EVA}^{[12,13]}$, de copolímero de butadieno - estireno (SBR)/EVA ${ }^{[14,15]}$, de borracha nitrílica (NBR)/EVA ${ }^{[16-18]}$ e $N B R /$ EPDM $^{[11,19]}$.

Além da compatibilização, o processo de vulcanização é de extrema importância do ponto de vista tecnológico e econômico, porque pode afetar significativamente as propriedades mecânicas e a resistência ao envelhecimento dos materiais elastoméricos. Tanto a densidade de reticulação como a sua distribuição em misturas elastoméricas dependem do tipo de acelerador, da razão entre acelerador e enxofre, da temperatura e tempo de reação e podem também ser afetadas pelo agente compatibilizante. De fato, copolímeros funcionalizados com grupos mercaptana, utilizados na compatibilização de vários sistemas elastoméricos heterogêneos, influenciaram, na maioria dos casos, os parâmetros de vulcanização ${ }^{[10]}$. No caso de misturas de NR/EPDM, a

Autor para correspondência: Bluma G. Soares, Instituto de Macromoléculas, UFRJ, Caixa Postal 68525, CEP: 21945-970, Rio de Janeiro, RJ, Brasil. 
adição de pequena porção de EPDM contendo grupos mercaptana (EPDMSH) acarretou em decréscimo ambos os tempos de segurança (scorch time) e tempo ótimo de cura, além de aumentar o torque máximo ${ }^{[10]}$.

Para avaliar melhor o efeito desses copolímeros funcionalizados no processo de vulcanização, resolveu-se estudar a cinética das reações envolvidas durante a vulcanização. As técnicas mais populares empregadas no estudo cinético da vulcanização de um elastômero incluem a calorimetria de varredura diferencial (DSC) e a reometria de disco oscilatório $(\mathrm{ODR})^{[20]}$. Essa última baseia-se no fato de que a densidade de reticulação é diretamente proporcional à dureza do material. A Figura 1 ilustra uma curva típica de torque $v s$. tempo, para um processo de vulcanização à base de enxofre na presença de acelerador, obtida a partir de experimentos no ODR. A primeira região é conhecida como período de indução, quando acredita-se que ocorra a maioria das reações envolvendo os aceleradores. A segunda região corresponde à cura propriamente dita, durante a qual a estrutura reticulada é formada. Na terceira região ocorre a maturação das reticulações, podendo haver um processo de reversão (degradação das ligações cruzadas), equilíbrio ou uma vulcanização adicional, porém lenta ${ }^{[21-22]}$.

O período que corresponde ao processo de cura pode ser descrito a partir de uma equação geral que relaciona o grau de conversão $(\alpha)$ em função do tempo que, para sistemas isotérmicos, é expresso da seguinte forma ${ }^{[21]}$ :

$$
\left(\frac{\partial \alpha}{\partial \mathrm{t}}\right)_{\mathrm{T}}=\mathrm{k}(\mathrm{T}) \mathrm{f}(\alpha)
$$

A função $k(T)$ pode ser expressa como uma equação de Arrhenius:

$$
k(T)=k_{0} e^{(-E / R T)}
$$

onde $k_{0}=$ fator pré-exponencial; $\mathrm{E}=$ energia de ativação; $\mathrm{R}=$ constante dos gases perfeitos; $\mathrm{e} \mathrm{T}=$ temperatura.

O segundo termo da Equação 1 é uma expressão matemática para o modelo cinético em função do grau de conversão e que pode assumir diferentes formas em função da ordem de reação de vulcanização (n):

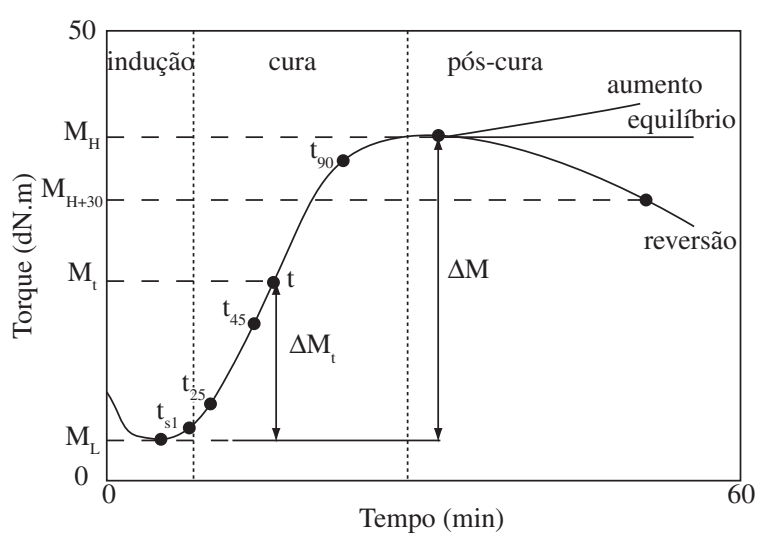

Figura 1. Curva típica de torque vs. tempo obtidas a partir do reômetro de disco oscilatório.

$$
f(\alpha)=(1-\alpha)^{n}
$$

A partir dos parâmetros de vulcanização obtidos do reômetro e da relação entre o grau de conversão com o tempo de vulcanização, vários modelos matemáticos baseados em aproximações fenomenológicas foram desenvolvidos, visando uma melhor compreensão do processo de vulcanização. Trabalhos excelentes de revisão foram publicados por Bateman e colaboradores $^{[23]}$, e mais recentemente por Krejsa e col$^{[24]}$.

Um esquema cinético simplificado foi proposto por Coran, há mais de 40 anos atrás, para a vulcanização com enxofre na presença de aceleradores ${ }^{[25]}$ :

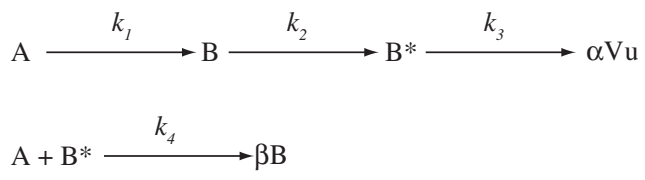

Onde A representa o acelerador e/ou seus produtos de reação com enxofre, $\mathrm{Zn}^{++}$, etc; B é o precursor das ligações cruzadas, ou seja, o produto da reação entre o agente sulfurante e a borracha; B* é a forma ativada de B, por exemplo, um radical livre polimérico do tipo sulfeto; Vu é a reticulação formada pela reação entre esses radicais livres poliméricos e cadeias da borracha; $\alpha$ e $\beta$ são parâmetros de ajuste estequiométricos; $k_{1}=$ constante de velocidade do consumo de acelerador; $k_{2}=$ constante de velocidade de formação do precursor de ligação cruzada; $k_{3}=$ constante de velocidade da formação de reticulações; e $k_{4}=$ constante de velocidade da formação de espécies inativas.

Outros métodos mais simples de determinação de parâmetros cinéticos foram posteriormente desenvolvidos por Yehia e Stoll ${ }^{[26]}$, e também por Chough e Chang ${ }^{[27]}$. Yehia e Stoll ${ }^{[26]}$ utilizaram o índice de velocidade de cura (CRI) baseado no tempo de segurança (scorch time) e no tempo ótimo de cura para descrever os parâmetros cinéticos enquanto que Chough e Chang ${ }^{[27]}$ utilizaram diferenças nos valores de torque em função do tempo.

O trabalho apresentado a seguir tem como objetivo estudar a influência do EPDM funcionalizado com grupos tioacetato (EPDMTA) ou com grupos mercaptana (EPDMSH) na cinética de vulcanização de misturas envolvendo NR/EPDM. Para esses estudos, foram adotados os modelos propostos por Coran ${ }^{[25]}$, por Yehia e Stoll ${ }^{[26]}$, e também por Chough e Chang ${ }^{[27]}$, a partir dos resultados obtidos do reômetro de disco oscilatório.

\section{Experimental}

\section{Materiais}

Borracha natural $(\mathrm{NR}),\left(\right.$ densidade $=0,864 \mathrm{~g} / \mathrm{cm}^{3}$, $\mathrm{Mn}=207.000$ e viscosidade Mooney $=87$ ) foi gentilmente cedida pela Michelin do Brasil. Terpolímero de etilenopropileno-dieno (EPDM 65$)(<\mathrm{Mn}>=150.000$; conteúdo de 
dieno $=114 \mathrm{mmol} / 100 \mathrm{~g}$; razão etileno $/$ propileno $=60 / 40$; viscosidade Mooney (ML $1+4$ a $\left.100{ }^{\circ} \mathrm{C}\right)=77$ ) foi gentilmente cedido por DSM Elastomeros do Brasil S. A. EPDM modificados com grupos tioacetato (EPDMTA) e mercaptana (EPDMSH) foram sintetizados em nossos laboratórios ${ }^{[8,9]}$. O esquema sintético bem como as estruturas de ambos são apresentados a seguir:

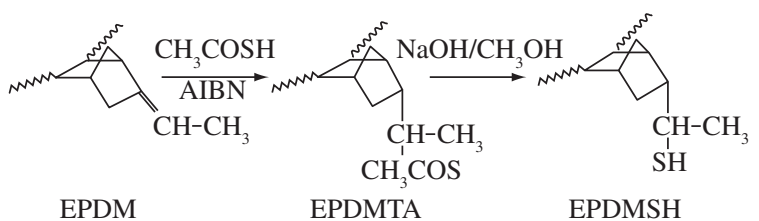

Óxido de zinco (Vetec), ácido esteárico (Vetec), Irganox 1010 (CIBA-GEIGY) e N-ciclohexil-2-benzotiazola sulfenamida, CBS (Bann Química Ltda) foram usados sem purificação.

\section{Preparação das misturas e medidas reométricas}

As misturas foram preparadas em misturador de cilindros (Berstorff) a $80{ }^{\circ} \mathrm{C}$ e $20 \mathrm{rpm} .70 \mathrm{phr}$ (part per hundred part of rubber, parte por cem partes de borracha) de $N R$ foram inicialmente mastigadas por $2 \mathrm{~min}$. Em seguida, $30 \mathrm{phr}$ de EPDM e 2,5 phr de EPDM funcionalizado (EPDMTA ou EPDMSH) foram adicionados. Após a homogeneização da mistura elastomérica (cerca de $4 \mathrm{~min}$ ), os agentes de cura foram adicionados na seguinte ordem: óxido de zinco (5 phr), ácido esteárico (1,5 phr), irganox 245 (1 phr), enxofre (2 phr) e N-ciclohexil-2-benzotiazola sulfenamida (CBS) (1 phr). O tempo de processamento após a adição de cada componente foi de cerca de 2 min.

Os parâmetros de vulcanização das misturas foram determinados a partir dos dados do reômetro de disco oscilatório (ODR) (Tecnologia Industrial, mol T100) com arco de $1^{\circ}$, de acordo com a norma ASTM D-2084/81. Os parâmetros cinéticos do processo de vulcanização foram calculados a partir de curvas de torque $v s$. tempo obtidas a partir de experimentos realizados a 150,160 e $170{ }^{\circ} \mathrm{C}$. A degradação das ligações cruzadas foi calculada a partir da taxa de reversão (R) utilizando a Equação 4:

$$
R(\%)=\frac{M_{H}-M_{H+30}}{M_{H}} \times 100
$$

onde $\mathrm{M}_{\mathrm{H}}$ é o torque máximo e $\mathrm{M}_{\mathrm{H}+30}$ é o torque observado após 30 min do torque máximo.

\section{Resultados e Discussão}

Os parâmetros de vulcanização das misturas compatibilizadas e não compatibilizadas obtidos a diferentes temperaturas são listados na Tabela 1. A Figura 2 ilustra as curvas de torque vs. tempo das misturas ensaiadas a $160{ }^{\circ} \mathrm{C}$. A adição de EPDMTA ou EPDMSH resultou em aumento no torque máximo e principalmente em um decréscimo significativo do tempo de segurança (scorch time).

A partir dos parâmetros de vulcanização obtidos a diferentes temperaturas, foi determinado o efeito da adição de copolímeros funcionalizados na cinética de vulcanização. Inicialmente, a cinética de vulcanização foi estimada a partir do índice de velocidade de cura (CRI), segundo o método relatado por Yehia e Stoll ${ }^{[26]}$, e definido pela Equação 5:

$$
C R I=\frac{1}{\left(t_{90}-t_{s l}\right)} \times 100
$$

onde: $\mathrm{t}_{90}$ é o tempo ótimo de vulcanização; e $\mathrm{t}_{\mathrm{s} 1} \mathrm{o}$ tempo de pré-vulcanização.

A dependência de CRI com o inverso da temperatura é apresentada na Figura 3. O CRI praticamente dobra com o aumento de $10{ }^{\circ} \mathrm{C}$ na temperatura de análise, mantendo a linearidade. Esse comportamento sugere uma tendência à cinética de primeira ordem. Comportamento semelhante foi também observado por Ehabe e colaboradores em sistemas de borracha natural vulcanizada com enxofre e aceleradores $^{[28]}$. De acordo com esse modelo, a presença do copolímero funcionalizado ocasionou um aumento na taxa de cura, sendo mais significativo para sistemas contendo EPDMSH.

Uma vez que o aumento do torque observado nos experimentos conduzidos no reômetro está diretamente relacionado

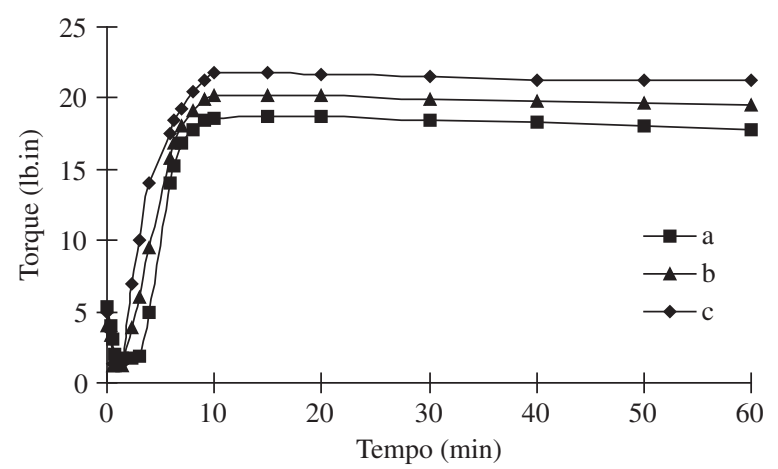

Figura 2. Dependência do torque $v s$. tempo de misturas NR/EPDM (70:30\%) a) sem copolímero funcionalizado e na presença de b) 2,5 phr de EPDMTA e c) 2,5 phr de EPDMSH.

\begin{tabular}{|c|c|c|c|c|c|c|c|c|c|c|c|c|}
\hline \multirow{2}{*}{$\begin{array}{c}\text { Copolímero } \\
\text { Funcionalizado }\end{array}$} & \multicolumn{4}{|c|}{$150{ }^{\circ} \mathrm{C}$} & \multicolumn{4}{|c|}{$160^{\circ} \mathrm{C}$} & \multicolumn{4}{|c|}{$170^{\circ} \mathrm{C}$} \\
\hline & $\begin{array}{c}\mathrm{M}_{\mathrm{L}}{ }^{\mathrm{a}} \\
\text { (N.m) }\end{array}$ & $\begin{array}{c}\mathrm{M}_{\mathrm{H}}{ }^{\mathrm{b}} \\
\text { (N.m) }\end{array}$ & $\begin{array}{c}\mathbf{t}_{\mathrm{s} 1}^{\mathrm{c}} \\
(\mathrm{min})\end{array}$ & $\begin{array}{c}\mathbf{t}_{90}{ }^{\mathrm{d}} \\
(\mathrm{min})\end{array}$ & $\begin{array}{c}M_{L}^{a} \\
\text { (N.m) }\end{array}$ & $\begin{array}{c}\mathbf{M}_{\mathrm{H}}{ }^{\mathrm{b}} \\
\text { (N.m) }\end{array}$ & $\begin{array}{c}\mathbf{t}_{\mathrm{s} 1}^{\mathrm{c}} \\
(\mathrm{min})\end{array}$ & $\begin{array}{c}\mathrm{t}_{90} \mathrm{~d}^{\mathrm{d}} \\
(\mathrm{min})\end{array}$ & $\begin{array}{c}\mathrm{M}_{\mathrm{L}}^{\mathrm{a}} \\
(\mathrm{N} . \mathrm{m})\end{array}$ & $\begin{array}{c}\mathbf{M}_{\mathrm{H}}{ }^{\mathrm{b}} \\
(\mathbf{N} . \mathbf{m})\end{array}$ & $\begin{array}{c}\mathbf{t}_{\mathrm{s} 1}^{\mathrm{c}} \\
(\mathrm{min})\end{array}$ & $\begin{array}{c}\mathbf{t}_{90}{ }^{d} \\
(\mathrm{~min})\end{array}$ \\
\hline Sem & 2,0 & 21,5 & 4,1 & 10,2 & 1,2 & 18,7 & 3,0 & 6,0 & 1,1 & 17,8 & 2,2 & 3,7 \\
\hline EPDMTA (2,5 phr) & 1,1 & 21,1 & 3,6 & 8,6 & 1,8 & 20,4 & 2,1 & 5,7 & 1,0 & 19,7 & 2,1 & 3,5 \\
\hline EPDMSH (2,5 phr) & 1,0 & 22,1 & 3,0 & 7,1 & 1,7 & 21,8 & 1,6 & 4,8 & 1,0 & 21,0 & 1,8 & 2,9 \\
\hline
\end{tabular}

Tabela 1. Efeito da adição de EPDM funcionalizado nos parâmetros de vulcanização das misturas NR/EPDM (70:30\%) a diferentes temperaturas.

${ }^{a}$ Torque mínimo; ${ }^{b}$ torque máximo; ${ }^{\mathrm{c}}$ tempo de segurança; $\mathrm{e}^{\mathrm{d}}$ tempo ótimo de cura. 
ao aumento na densidade de reticulação ${ }^{[29]}$, resolveu-se determinar as constantes cinéticas de vulcanização das misturas, utilizando o modelo proposto por Chough e Chang ${ }^{[27]}$. Esse modelo assume que a vulcanização segue uma cinética de primeira ordem. Para tanto, o comportamento cinético foi avaliado a partir de mudanças no torque compreendido entre os valores correspondentes a 25 e $45 \%$ de conversão, uma vez que nesse intervalo, a cinética de primeira ordem é garantida. A constante de velocidade foi calculada a partir da Equação 6:

$$
\ln \frac{M_{H}-M_{L}}{M_{H}-M_{t}}=k t \text { ou seja } \ln \frac{M_{45}-M_{25}}{M_{45}-M_{t}}=k t
$$

onde $M_{H}$ e $M_{L}$ são os torques máximo e mínimo, respectivamente, $M_{45}$ e $M_{25}$ correspondem aos torques a 45 e $25 \%$ de conversão, respectivamente e $M_{t}$ representa o torque a um tempo t. A Tabela 2 apresenta os valores das constantes de velocidade para cada temperatura estudada, bem como a energia de ativação do processo de vulcanização em função da presença de EPDMTA ou EPDMSH. A energia de ativação foi calculada utilizando a equação de Arrhenius (Equação 2).

Observa-se que, para todas as temperaturas estudadas, a presença dos copolímeros funcionalizados acarretou um aumento nos valores de $\mathrm{k}$, sendo esse efeito mais pronunciado para misturas contendo EPDMSH. Nota-se também que a energia de ativação é menor para as misturas contendo EPDMTA ou EPDMSH, confirmando a capacidade dos grupos tioacetato e mercaptana em atuarem como acelerador no processo de vulcanização à base de enxofre.

Embora os modelos discutidos até agora forneçam informações importantes a respeito da influência dos copolímeros contendo grupos tioacetato ou mercaptana na velocidade de cura, eles não conseguem distinguir o estágio do processo onde a sua atuação é mais efetiva. Resolveu-se, então, utilizar o modelo proposto por Coran ${ }^{[25]}$. Como foi discutido na Introdução desse trabalho, esse modelo é capaz de distinguir as diferentes etapas que compõem o processo, sendo capaz de determinar as constantes de velocidade de algumas dessas etapas. Para a determinação das constantes de velocidade se-

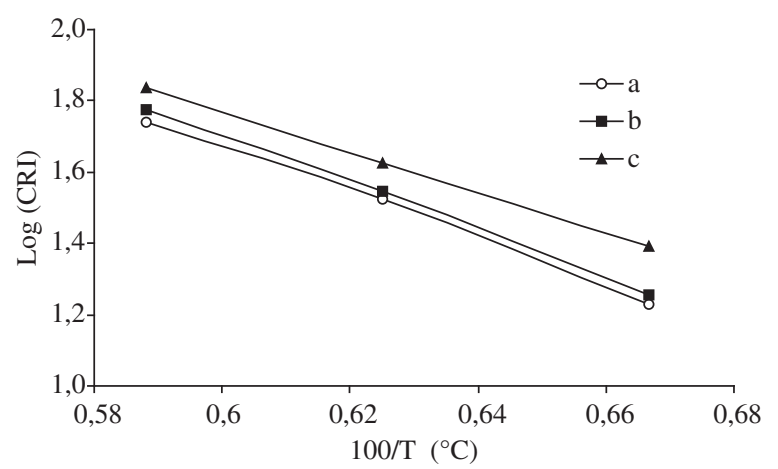

Figura 3. Dependência de CRI em função do inverso da temperatura, para misturas NR/EPDM (70:30\%) (a) sem copolímero funcionalizado e na presença de (b) 2,5 phr de EPDMTA e (c) 2,5 phr de EPDMSH. gundo o modelo de Coran, a variação do torque foi colocada em função do tempo, segundo a expressão a seguir:

$$
\ln \left(1-\frac{\Delta M_{t}}{\Delta M}\right)=k t
$$

onde $\Delta M$ representa a diferença entre o torque máximo $\left(\mathrm{M}_{\mathrm{H}}\right)$ e o torque mínimo $\left(M_{L}\right)$ e $\Delta M_{t}$ representa a diferença entre o torque a um tempo te o torque mínimo. A aplicação da Equação 7 na avaliação do efeito dos copolímeros funcionalizados (EPDMTA e EPDMSH) em misturas NR/EPDM é ilustrada na Figura 4. Para esse estudo, foram selecionados os resultados torque obtidos na temperatura de vulcanização igual a $160{ }^{\circ} \mathrm{C}$ porque nessa temperatura, as diferenças entre os sistemas modificados e não modificado foram mais significativas. A partir dessas curvas, foram calculados os parâmetros cinéticos listados na Tabela 3 .

A constante de velocidade $\mathrm{k}_{2}$ corresponde ao coeficiente angular da reta, cuja faixa de conversão segue a cinética de primeira ordem ${ }^{[30,31]} \cdot t_{\text {dis }}$ (tempo necessário para que a reação seja considerada de primeira ordem) corresponde ao ponto onde termina a curvatura do gráfico de $\ln \left(1-\Delta \mathrm{M}_{\mathrm{t}} / \Delta m\right) v s$. tempo (Figura 4). $t_{i}$ é o tempo correspondente à interseção das duas regiões desse gráfico e é considerado o tempo de indução ${ }^{[25]}$. A constante de velocidade $k_{l}$, que determina o período de indução, é calculado segundo a expressão:

Tabela 2. Efeito dos copolímeros funcionalizados nos parâmetros cinéticos das misturas NR/EPDM (70: 30\%), calculados segundo o

\begin{tabular}{|c|c|c|c|c|}
\hline & \multicolumn{3}{|c|}{$k^{\mathrm{a}}(\min .)^{-1}$} & \multirow{2}{*}{$\begin{array}{c}E a^{\mathbf{b}} \\
(\mathrm{KJ} / \mathrm{mol})\end{array}$} \\
\hline & $150{ }^{\circ} \mathrm{C}$ & $160^{\circ} \mathrm{C}$ & $170{ }^{\circ} \mathrm{C}$ & \\
\hline sem copolímero & 0,569 & 0,824 & 1,325 & 66,48 \\
\hline EPDMTA $(2,5 \mathrm{phr})$ & 0,755 & 1,179 & 1,674 & 62,05 \\
\hline EPDMSH (2,5 phr) & 0,885 & 1,553 & 1,795 & 55,32 \\
\hline
\end{tabular}
modelo de Chough e Chang.

${ }^{\mathrm{a} C}$ Constante de vulcanização; ${ }^{\mathrm{b}}$ Energia de ativação.

Tabela 3. Parâmetros cinéticos determinados a $160^{\circ} \mathrm{C}$, segundo o modelo de Coran, para misturas NR/EPDM em função da compa-

\begin{tabular}{|c|c|c|c|}
\hline \multirow{2}{*}{$\begin{array}{l}\text { Parâmetros } \\
\text { cinéticos }\end{array}$} & \multicolumn{3}{|c|}{ Mistura NR/EPDM (70:30\%) } \\
\hline & $\begin{array}{c}\text { Sem } \\
\text { compatibilizante }\end{array}$ & $\begin{array}{l}\text { EPDMTA } \\
(2,5 \text { phr })\end{array}$ & $\begin{array}{c}\text { EPDMSH } \\
(2,5 \text { phr })\end{array}$ \\
\hline$t_{d i s}^{a}$ & 3,20 & 2,59 & 2,24 \\
\hline$t_{i}^{b}$ & 2,96 & 2,43 & 2,00 \\
\hline$t_{d i s}-\mathrm{t}_{\mathrm{i}}$ & 0,68 & 0,16 & 0,24 \\
\hline$k_{1}$ & 1,35 & 1,46 & 3,21 \\
\hline$k_{2}$ & 0,917 & 0,887 & 0,945 \\
\hline$k_{4} / k_{3}$ & 19.442 & 16.737 & 18.629 \\
\hline $\begin{array}{l}\text { Taxa de } \\
\text { reversão (\%) }\end{array}$ & 3,67 & 0,87 & 1,43 \\
\hline $\begin{array}{l}\text { a tempo de displa } \\
\text { siderada de prim } \\
\text { velocidade do co } \\
\text { de formação do } \mathrm{p} \\
\text { cidade da formaç } \\
\text { da formação de e }\end{array}$ & $\begin{array}{l}\text { y - (tempo necessário } \\
\text { eira ordem); }{ }^{\text {b }} \text { tempo } \\
\text { nsumo de acelerador; } \\
\text { recursor de ligação cr } \\
\text { ão de reticulações; and } \\
\text { spécies inativas. }\end{array}$ & $\begin{array}{l}\text { para que a re } \\
\text { indução; } k_{l}=\end{array}$ & $\begin{array}{l}\text { ção seja con- } \\
=\text { constante de } \\
\text { de velocidade } \\
\text { tante de velo- }\end{array}$ \\
\hline
\end{tabular}
tibilização. 


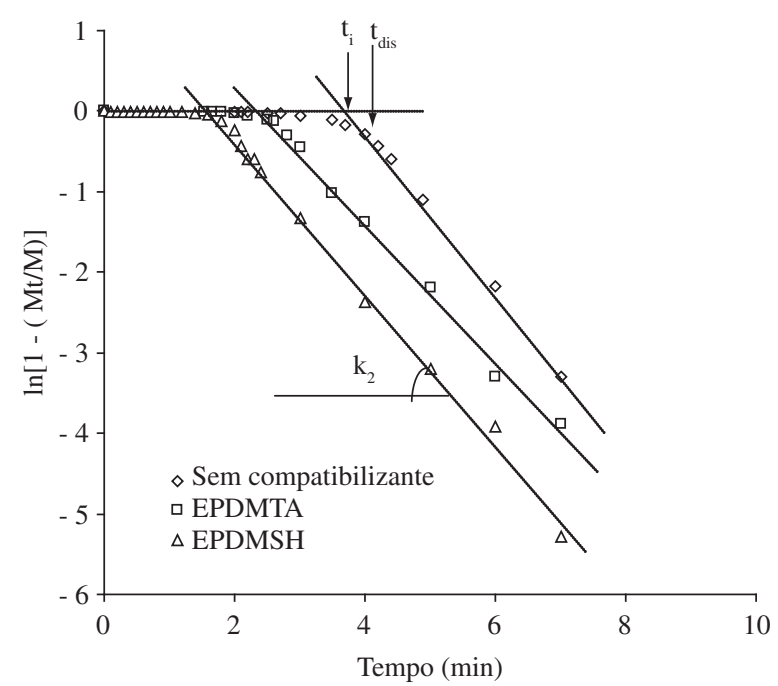

Figura 4. Dependência de $\mathrm{h}(1-\Delta \mathrm{M} / \Delta \mathrm{M})$ vs. tempo, para misturas NR/EPDM (70:30) a) sem copolímero funcionalizado e na presença de b) 2,5 phr de EPDMTA e c) 2,5 phr de EPDMSH.

$$
k_{1} t_{i}-\ln k_{1}=k_{2} t_{d i s}-\ln k_{2}
$$

As constantes $k_{3}$ e $k_{4}$ não podem ser calculadas separadamente. Todavia, a razão $k_{4} / k_{3}$ pode ser determinada a partir da Equação 9 ${ }^{[32]}$ :

$$
\frac{k_{4}}{k_{3}}=-\left(\frac{M_{A} U}{C_{A}}\right)\left(\frac{\Delta M}{\Delta M_{T}}\right) \ln \left(\frac{k_{2} e^{k_{1} t}-k_{l} e^{k_{2} t}}{k_{2}-k_{I}}\right)
$$

Onde $k_{4} / k_{3}$ é uma razão adimensional de constante de velocidade; $\mathrm{C}_{\mathrm{A}}$ é a concentração do acelerador expressa em phr (partes por cem partes de borracha); $M_{A}$ é a massa molar do acelerador; e $U$ é o número de moles de ligações duplas por $100 \mathrm{~g}$ de borracha. Nas misturas NR/EPDM (70:30), esse número $U$ corresponde a 0,73 moles/100 g, calculado a partir de ressonância magnética nuclear. A razão $k_{4} / k_{3}$ é conhecida como razão de extinção e indica a tendência do acelerador ou de um produto formado nos primeiros estágios da reação, de inibir a reticulação.

Como observado na Figura 4 e Tabela 3, o tempo $t_{\text {dis }}$ (tempo necessário para que a reação seja considerada de primeira ordem) e $t_{i}$ (tempo de indução) diminuem para as misturas compatibilizadas. Isso ocasiona maiores valores de $k_{1}$. O maior valor dessa constante foi observado para misturas contendo EPDMSH, o que indica maior rapidez em que o acelerador e/ou seus produtos de reação com enxofre são convertidos em B, o precursor das ligações cruzadas. Esses resultados confirmam a atuação do EPDMSH na primeira etapa do processo de vulcanização, provavelmente devido a possíveis interações entre os grupos mercaptan do copolímero e os aceleradores utilizados, aumentando a sua velocidade de consumo. Sugere-se, então, que EPDMSH esteja agindo como um acelerador secundário. EPDMTA também aumenta a velocidade da primeira etapa, mas segundo os valores de $\mathrm{k}_{1}$ apresentados na Tabela 3 , esse efeito é pouco significativo.
Após o período de indução, o processo de conversão das espécies B em B* (forma ativada de B, ou seja, um radical livre polimérico do tipo sulfeto) caracterizado pela constante $\mathrm{k}_{2}$, não é afetado pela presença dos copolímeros funcionalizados. Apenas um ligeiro acréscimo de $\mathrm{k}_{2}$ é observado para misturas com EPDMSH.

Entretanto, a razão $k_{4} / k_{3}$ apresentou mudanças significativas com a presença dos copolímeros, sendo maior com EPDMTA. Essa razão reflete a competição entre o processo de vulcanização $\left(k_{3}\right)$ e a reação entre $\mathrm{B} *$ e os aceleradores dando origem ao precursor de ligações cruzadas $\left(k_{4}\right)$. Quanto menor o valor dessa razão maior será a tendência à reticulação. Pode-se sugerir, a partir dos valores de $k_{4} / k_{3}$, que EPDMTA facilita o processo de reticulação, reduzindo a possibilidade de reação reversa. Misturas com EPDMSH, por apresentar maior velocidade de formação de B (maior $k_{l}$ ) favorece também o encontro de B* com A (processo envolvendo $k_{4}$ ), retardando um pouco o processo de reticulação propriamente dito. É importante enfatizar, entretanto que, mistura não compatibilizada apresentou maior período de indução e também maior valor da razão $k_{4} / k_{3}$.

Com relação ao processo de decomposição das reticulações medido a partir da taxa de reversão (R), observa-se uma grande tendência à reversão para misturas não compatibilizadas. Essa tendência decresce com a presença de EPDMSH, tornando-se bem menor quando se utiliza EPDMTA como agente compatibilizante.

\section{Conclusões}

EPDM funcionalizado com grupos tioacetato (EPDMTA) e mercaptana (EPDMSH) atuaram como aceleradores do processo de vulcanização das misturas NR/EPDM (70:30) na presença de enxofre e CBS, sendo EPDMSH mais efetivo do que EPDMTA pelos valores de CRI e pelo modelo de Chough e Chang. Entretanto, ao utilizar o modelo de Coran, observou-se que EPDMTA e EPDMSH atuam em distintas etapas da vulcanização: EPDMSH atua preferencialmente no início do processo de vulcanização quando são formados os precursores da ligação cruzada enquanto que EPDMTA aumenta a velocidade da formação das reticulações propriamente dita. Além disso, EPDMTA é mais efetivo do que EPDMSH em diminuir a chance de decomposição das ligações cruzadas através do processo de reversão. Esses resultados associados à atuação interfacial desses copolímeros contribuem para o desenvolvimento de misturas NR/EPDM com propriedades mecânicas superiores e melhor resistência ao envelhecimento, com o relatado em artigos anteriores ${ }^{[9,10]}$.

\section{Agradecimentos}

Os autores agradecem ao Conselho Nacional de Desenvolvimento Científico e Tecnológico (CNPq), Coordenação de Aperfeiçoamento de Pessoal de Nivel Superior (CAPES), 
Financiadora de Estudos e Projetos (FINEP) e Fundação de Amparo à Pesquisa do Estado do Rio de Janeiro (FAPERJ), pelo suporte financeiro ao projeto.

\section{Referências Bibliográficas}

1. Hess, W. M.; Herd, C. R. \& Vegvari, P. C. - Rubber Chem. Technol., 66, p. 329 (1993).

2. Woods, M.E.\&DavidsonJ.A.-RubberChem. Technol.,49, p. 112 (1976).

3. Chang, Y. W.; Shin, Y. S.; Chun, H. \& Nah, C. - J. Appl. Polym. Sci., 73, p. 749 (1999).

4. Baranwal, K. C. \& Son, P. N. - Rubber Chem. Technol., 47, p. 88 (1974).

5. Coran, A. Y. - Rubber Chem. Technol., 61, p. 281 (1988).

6. Coran, A. Y. - Rubber Chem. Technol., 64, p. 801 (1991).

7. Suma, N.; Joseph, R. \& Francis, D. J. - Kautsch Gummi Kunstst., 43, p. 1095 (1990).

8. Oliveira,M.G.; Soares,B.G.;Santos,C.M.F.;Diniz,M.F.\& Dutra, R. C. L. - Macromol. Rapid. Commun., 20, p. 526 (1999).

9. Sirqueira, A. S. \& Soares, B. G. - J. Appl. Polym. Sci., 83, p. 2892 (2002).

10.Sirqueira, A. S. \& Soares, B. G. - Eur. Polym. J., 39, p. 2283 (2003).

11.Soares, B. G.; Sirqueira, A. S.; Oliveira, M. G. \& Almeida, M. S. M. - Macromol. Symp., 186, p. 45 (2002).

12.Jansen, P.; Amorim, M.; Gomes, A. S. \& Soares, B. G. - J. Appl. Polym. Sci., 58, p. 101 (1995).

13.Jansen,P.; Gomes,A.S.\&Soares,B.G.-J.Appl.Polym.Sci., 61, p. 591 (1996).

14. Soares, B. G.;Alves, F. F.; Oliveira, M. G.; Moreira, A. C.F.; Garcia, F. G. \& Lopes, M. F. S. - Eur. Polym. J., 37, p. 1577 (2001).

15.Soares, B. G.;Alves, F.F.; Oliveira, M. G. \& Moreira,A.C.F. - J. Appl. Polym. Sci., 86, p.239 (2002).
16.Jansen, P. \& Soares, B. G. - J. Appl. Polym. Sci., 79, p. 193 (2001)

17.Jansen, P. \& Soares, B. G. - J. Appl. Polym. Sci., 84, p. 2335 (2002).

18.Jansen, P.; Garcia, F. G. \& Soares, B. G. - J. Appl. Polym Sci., 90, p. 2391 (2003).

19. Oliveira, M. G. \& Soares, B. G. - J. Appl. Polym. Sci., 91, p. 1404 (2004).

20.Akiba, M. \& Hashim, A. S. - Prog. Polym. Sci., 22, p. 475 (1997).

21.Chan, T. W.; Shyu, G. D. \& Isayev, A. I. - Rubber Chem. Technol., 66, p. 849 (1993).

22.Isayev, A. I. \& Deng, J. S. - Rubber Chem. Technol., 61, p. 340 (1988).

23. Bateman, L.; Moore, C. G.; Porter, M. \& Saville, B. - "In The Chemistry and Physics of Rubber-like Substances", cap 15, L. Bateman (ed.), Maclaren and sons Ltd., London (1963).

24.Krejsa, M.R.; Koenig,J.L. \&Sullivan,A.B.-RubberChem. Technol., 67, p. 348 (1994).

25.Coran, A. Y. - Rubber Chem Technol., 37, p. 689 (1964).

26. Yehia, A. A. \& Stoll, B. - Kautsch Gummi Kunsts., 40, p. 950 (1987).

27.Chough, S. H. \& Chang, D. H. - J. Appl. Polym. Sci., 61, p. 449 (1996).

28.Ehabe, E. E. \& Farid, S. A. - Eur. Polym. J., 37, p. 329 (2001).

29.Cotten, G. R. - Rubber Chem. Technol., 45, p. 129 (1972).

30.Duchácek, V. - J. Appl. Polym. Sci., 18, p. 125 (1974).

31.Duchácek, V. - J. Appl. Polym. Sci., 22, p. 227 (1978).

32.Coran, A. Y. - Rubber Chem. Technol., 38, p. 1 (1965).

Enviado: 27/04/06

Reenviado: 29/08/06

Aceito: 05/09/06 